\title{
Commentary on "Measuring Aksak Rhythm and Synchronization in Transylvanian Village Music by Using Motion Capture"
}

\author{
JÉRÔME CLER \\ Université Paris-Sorbonne
}

\begin{abstract}
While the methodologies employed by Bonini-Baraldi et al., document the rhythms produced in a single set of performances, those methodologies would be more relevant if applied to a comparative study of various aksak rhythms, rather than to a study of a single rhythmic pattern. The results of the present study are not very different from the findings of R.I.T.M.O seminar (J. During, J. Cler. et al,) during the mid 1990s.
\end{abstract}

Submitted 2015 June 3; accepted 2015 September 15.

KEYWORDS: Aksak rhythm, Isochronic pulse, Cross-cultural studies

MANY works in ethnomusicology since the 1990s have focused on rhythm typologies in oral traditions and on the specific issues of aksak rhythm, rhythmic irregularity, an-isochronic pulses, and their cognitive aspects. With the support of the Société Française d'Ethnomusicologie, in the 1990s "RITMO" seminar ("Rythme et Image du Temps dans la Musique Orientale") was led by Jean During. During this seminar we conducted a number of experiments, for example, with Turkish and Balutch masters, or with oriental drummers. Results of some of these experiments were published in a volume of the Cahier de Musiques Traditionnelles, issue $\mathrm{n}^{\circ}$ 10, 1997, ("Rythmes"), where Jean During created his concept of "Ovoïd Rhythm", and where Jacques Bouet questioned the problem of non-isochronous pulses in Transylvanian music. In the same volume my article, "Aksak: les catastrophes d'un modèle", questioned the relevance of the arithmetic conception of 2 and 3 subpulse units, as there seemed to be unequal blocks of duration in the music we studied, blocks conceived as continuous rather than discrete or subdivided into series of isochronous units. Jean During's theory of ovoïd metre had many of the same characteristics, but the main difference was that in aksak the isochronic pulse was still present, as an implicit reference, while in ovoïd rhythms the durations could not be reduced to an underlying pulse, or an arithmetic logic. We both examined the mean ratios of the unequal durations to compare various segments of the musics we studied. Our method was empirical, and the experiments were mostly inspired by concrete situations with the musicians who participated in our study. One of the most interesting set of experiments involved crosscultural tests: could a Tadjik musician identify/classify a turkish rhythm that doesn't exist in Tadjik culture, or how a Balutch musician would react to isochronous or metronomic aksak rhythms that looked like the specific Balutch rhythm we wanted to analyse or understand?

The aim of such studies was not only the analysis of musical rhythm in various oriental cultures, but also to define its rhythmic habitus: aksak is not only a specific type of Balkan or Turkish rhythm, it is first of all a basic rhythmic schema, under which local musicians compose Long and Short durations, where the fundamental L:S ratio=1.5. Regarding aksak in particular, the main question was how can one align this schematic conception with the necessary existence in some musical contexts of groups of 2 or 3 isochronous units $(2+3)$ ? In Bulgarian and Turkish musics, melodic articulations confirm that these isochronous units can be relevant to describe the metric structure, even if a non-isochronous pulse was explicitly present for the musicians and the reference for their beat (e.g., hand claps). One other aspect of this analysis involved tempo: in the Turkish music we studied, there was a large range of tempos for each metrical structure (isochronous pulses from 35 to $450 \mathrm{BPM}$ ), and in three distinct classes of tempo in the representation of the musicians. Our conclusions from these preliminary studies of aksak rhythm were as follows:

- The structuring of the rhythm by a Long/Short distinction is the rhythmic basis for the local musicians, but the isochronous subdivision is also relevant for the analyst, and also exists as a 
subconscious structuration of the rhythm. The supposed distinction between $9 / 16$ and 5/4, which was the main focus of my article, was not evident after having measured the durations in various performances. This fact inspired the idea that there is a conflict between two logics, or two cognitive models (i.e., isochronous subpulses versus categorically distinct long vs. short duration.

- There is a distinction between surface rhythms and underlying beat structures. For example, the 4 beat pattern of $2+2+2+3$ (played with lute) is actually performed as a 3 element rhythm $(4+2+3$, with davul-zurna).

Beyond the analytic point of view, and the dilemma of continuous versus discrete conceptions of rhythm (blocks of duration versus underlying isochronous pulses), what is actually questioned is the habitus that produces such rhythms. For example, contrametricity and commetricity, as Kolinski defines them, are not only descriptive concepts, but rhythmical behaviours that commands the cultural production of rhythmic structures (see Cler 2010).

This is why, for me, in this context and after those previous works, Bonini-Baraldi et al.'s paper is important as a first step to a wider exploration of aksak structures. Especially because the experimental tools motion capture are here very efficient. I will comment only the first study included in their paper, concerning aksak's durational structure. Their experimental process includes 3 agents: the musicians (who served as the experimental subjects), the "expert" musician/ethnomusicologist who specializes in this music, and the music psychologist. It is important to insist on the second agent, expert musician/researcher, who can have the function of an interface between the musicians from the field, and the analytic results of the study. Thus in some ways the experiment here is not so different from those made in the 1990's with Jean During, except for the use of motion capture. In those experiments there always was a transitive process between the musician, the expert/interface (who could be also the musician), and the objective of our measurements.

Bonini-Baraldi's article follows Brăiloiu's definition of aksak: a rhythm is an aksak when: (1) it is periodic and (2) each period is made up of the combination of two categorically distinct duration units (Short $=\mathrm{S}$ and Long $=\mathrm{L}$ ), nominally in a ratio of $\mathrm{S}: \mathrm{L}=2: 3$. It is important to recall that Brăiloiu had brought a new conception of this kind of rhythms, insisting on the concept of bichronicity (two units), instead of the Bartokian "Bulgarian Rhythm," where the aksak units were subdivided in isochronic units (3s or $2 \mathrm{~s}$ ). This bichronal approach is related to "elasticity," that various aksak rhythms are subject to timing variations, such that we cannot certify that their underlying ratio is centered around 3:2. And how can this be determined from the timing data, if the subdividing isochronous unit is never explicit? The main theoretical problem can thus be formulated in these terms : how can we chose between a continuous way of conceiving the constitutive durations, or a discrete subdivision of these durations in terms of isochronous minimal units (or even in an-isochronous pulses with some strict ratio relationship, such as $\mathrm{S}=1 \mathrm{~L}=1,5$, or $\mathrm{S}=1, \mathrm{~L}=1,33$ etc.). The real issue seems to rely in the idea that we have to quit the arithmetic explanations based on natural number ratios and to take in consideration broader "topological" explanations--for example, as mentioned above, relating various ratios to melodic contour or articulation, rather than with quantities of subdivision units. In my own work (Cler 1997) I have also examined this problem, and have suggested that there are two kinds of aksak, one with underlying explicitation of the isochronous unit, the other one without, depending on the tempo.

I would like to further examine another theoretical aspect of aksak bichronicity: the fact that "swinging" (i.e., adding expressive timing) a ratio of 1:2 can produce 2:3, 3:4 ratio, or even more subtle ratio. The Transylvanian cases studied here suggest parallels to Jean During's "Ovoïd rhythms," where there is no "ratio", but the concatenation of real units in themselves, units that are largely linked with movement and gesture. In Bonini-Baraldi's study those units would be the bow movements, while in During's study of Balutch or Tadjik the "ovoïds" involved the right hand movement up and down the strings. That is, why should we need to refer to aksak logic? In the aksak logic (i.e., that proposed by Brăiloiu'), we have necessarily at the same time 2 blocks of duration, and an underlying isochronous pulsation that gives the durations their. consistency. I believe that in the case of aksak "a la Turca" the brain also counts, or follows the isochronous pulse inside the non-isochronous structure, which is conceptually prior. At that point it is necessary to observe, in the field, how the rhythm is perceived or created: musicians and listeners both tap the beat by following this "limping pulse" (i.e. they tap the $\mathrm{S}$ and the $\mathrm{L}$ at each period).

For Bonini-Baraldi and his colleagues' future work, I have a number of questions:

- In this culture, is the same rhythm performed at various tempos and/or genres, so that we could extend their experimental method to other forms? 
- Is the same mean S:L ratio (and range of ratios) present in other forms (as in the Purtata de Frata anaysed by Jacques Bouet), and is it possible to confirm the same type of irregularity/inequality from one form to another?

- How would these musicians perform aksak in musical genres and styles from other cultures, as the 9/16 vs 5/4 variants that even Simha Arom was not able to distinguish?

- Isn't there another type of analysis dealing with the topology of aksak rhythms, (versus arithmetic approaches)?

Finally, while precise measures of the S:L proportions in live performances may help us to understand how musicians conceive the aksak in different parts of the world, for the music studied by Bonini-Baraldi and his colleagues it also seems to me very important to share the experimental results with various musicians. For it is very significant that the authors, after having described all the process of their experimentation and measurement, go back to the simple ethnographic observation regarding the "co-presence of two different beat classes" (p. 8). And thus the authors conclude: "the Transylvanian slow aksak is not bodily decomposed in smaller pulses." (p. 8). Thus experimental measurements using improved methods such as motion capture, will continued to require supplementation from old-school, practical and fieldworkinspired experience.

\section{NOTES}

[1] Correspondence can be addressed to: Prof J. Cler, U.F.R. De Musique et Musicologie, Université ParisSorbonne, jerome.cler@paris-sorbonne.fr .

\section{REFERENCES}

Bachir, T. (2007). L'ethnomusicologie, son identité, ses modes d'emploi. Cahiers d'Ethnomusicologie 20, 293-298.

Bouët, J. (1997). Pulsations retrouvées. Les outils de la réalisation rythmique avant l'ère du métronome. Cahiers de Musiques Traditionnelles, 10, 107-125.

Cler, J. (1997). Aksak: les catastrophes d'un modèle. Cahiers de Musiques Traditionnelles, 10, 60-80.

Cler, J. (2010). Rhythmos, skhèma: pour une typologie des rythmes en tradition orale. In Rythmes de L'homme, Rythmes du Monde, C. Doumet \& A.W. Lasovksi, eds. Paris: Hermann.

During, J. (1997). Rythmes ovoïdes et quadrature du cycle. Cahiers de Musiques Traditionnelles, 10, 1736. 